\title{
EUS-guided intrahepatic biliary drainage: a large retrospective series and subgroup comparison between percutaneous drainage in hilar stenoses or postsurgical anatomy
}

\section{(ㄷ)(1) $(2) \Theta$}

Authors

Giuseppe Vanella ${ }^{1,2}$, Michiel Bronswijk ${ }^{1}$, Geert Maleux ${ }^{3}$, Hannah van Malenstein ${ }^{1}$, Wim Laleman ${ }^{1}$, Schalk Van der Merwe $^{1}$

Institutions

1 Department of Gastroenterology and Hepatology, University Hospital Gasthuisberg, University of Leuven, Leuven, Belgium

2 Pancreatobiliary Endoscopy and Endosonography Division, IRCSS San Raffaele Scientific Institute, Milan, Italy

3 Department of Interventional Radiology, University Hospital Gasthuisberg, University of Leuven, Leuven, Belgium

\section{Bibliography}

Endoscopy International Open 2020; 08: E1782-E1794

DOI 10.1055/a-1264-7511

ISSN 2364-3722

(C) 2020. The Author(s).

This is an open access article published by Thieme under the terms of the Creative Commons Attribution-NonDerivative-NonCommercial License, permitting copying and reproduction so long as the original work is given appropriate credit. Contents may not be used for commecial purposes, or adapted, remixed, transformed or built upon. (https://creativecommons.org/licenses/by-nc-nd/4.0/)

Corresponding author

Schalk van der Merwe MD, PhD, Department of Gastroenterology and Hepatology, University Hospital

Gasthuisberg, University of Leuven, Herestraat 49,

B - 3000 Leuven, Belgium

Fax: + 3216344387

schalk.vandermerwe@uzleuven.be

$\circledast$ Supplementary material is available under https://doi.org/10.1055/a-1264-7511

\section{ABSTRACT}

Background and study aims Endoscopic ultrasoundguided intrahepatic biliary drainage (EUS-IBD) struggles to find a place in management algorithms, especially compar- ed to percutaneous drainage (PTBD). In the setting of hilar stenoses or postsurgical anatomy data are even more limited.

Patients and methods All consecutive EUS-IBDs performed in our tertiary referral center between 2012-2019 were retrospectively evaluated. Rendez-vous (RVs), antegrade stenting (AS) and hepatico-gastrostomies (HGs) were compared. The predefined subgroup of EUS-IBD patients with proximal stenosis/surgically-altered anatomy was matched 1:1 with PTBD performed for the same indications. Efficacy, safety and events during follow-up were compared.

Results One hundred four EUS-IBDs were included (malignancies $=87.7 \%$ ). These consisted of 16 RVs, 43 ASs and 45 HGs. Technical and clinical success rates were $89.4 \%$ and $96.2 \%$, respectively. Any-degree, severe and fatal adverse events (AEs) occurred in $23.3 \%, 2.9 \%$, and $0.9 \%$ respectively. Benign indications were more common among RVs while proximal stenoses, surgically-altered anatomy, and disconnected left ductal system among HGs. Procedures were shorter with HGs performed with specifically designed stents ( 25 vs. 48 minutes, $P=0.004$ ) and there was also a trend toward less dysfunction with those stents $(6.7 \% \mathrm{vs}$. $30 \%, P=0.09$ ) compared with previous approaches. Among patients with proximal stenosis/surgically-altered anatomy, EUS-IBD vs. PTBD showed higher rates of clinical success (97.4\% vs. $79.5 \%, P=0.01$ ), reduced post-procedural pain ( $17.8 \%$ vs. $44.4 \%, p=0.004)$, shorter median hospital stay (7.5 vs 11.5 days, $P=0.01$ ), lower rates of stent dysfunction ( $15.8 \%$ vs. $42.9 \%, P=0.01$ ), and the mean number of reinterventions was lower (0.4 vs. $2.8, P<0.0001)$.

Conclusions EUS-IBD has high technical and clinical success with an acceptable safety profile. HGs show comparable outcomes, which are likely to further improve with dedicated tools. For proximal strictures and surgically-altered anatomy, EUS-IBD seems superior to PTBD. 


\section{Introduction}

Endoscopic retrograde cholangiopancreatography (ERCP) is the first-line treatment modality for biliary drainage (BD). However, cannulation and stenting may fail in up to $10 \%$ of cases [1]. Moreover, in the setting of surgically-altered anatomy a retrograde approach can be arduous or not feasible [2]

Until recently, the only established alternative was percutaneous transhepatic BD (PTBD), but developments in therapeutic endoscopic ultrasound (EUS) have radically expanded the possibilities for accessing the biliary tree in case of failed ERCP [3].

Evidence has been accumulating regarding the use of EUSguided BD (EUS-BD) in the management of extrahepatic biliary obstruction (e.g. EUS-guided choledochoduodenostomy [eCD]) [4-6]. However, the use of EUS-guided BD with transgastric access for rendez-vous (RV), antegrade stenting (AS) or hepaticogastrostomy (HG), has been less well explored and is often perceived to be riskier or less effective $[7,8]$. Comparisons of EUS-BD to PTBD are usually restricted to distal obstructions and evidence is lacking on alternatives to PTBD, when extrahe- patic biliary drainage is not feasible, such as in the case of hilar/ intrahepatic stenoses or surgically-altered anatomy. Finally, RV, AS, and HG have never been compared, nor have outcomes with different devices. For all these reasons, EUS-guided intrahepatic BD (EUS-IBD) struggles to find a well-defined role in clinical algorithms.

Our primary aim was to retrospectively analyze efficacy and safety of EUS-IBDs performed for any indication in a tertiary referral center over 8 years. Indications and outcomes with three different techniques were also separately described. Our secondary aim was to compare the efficacy and safety of EUSIBDs performed in a prespecified subgroup of patients with hilar/intrahepatic stenoses or postsurgical anatomy, matched 1:1 with PTBDs executed for the same indications.

\section{Patients and methods}

A retrospective search of Leuven University Hospitals electronic database was performed to identify all consecutive EUS-IBDs executed between 2012 and 2019. All EUS-IBD cases were eligi-

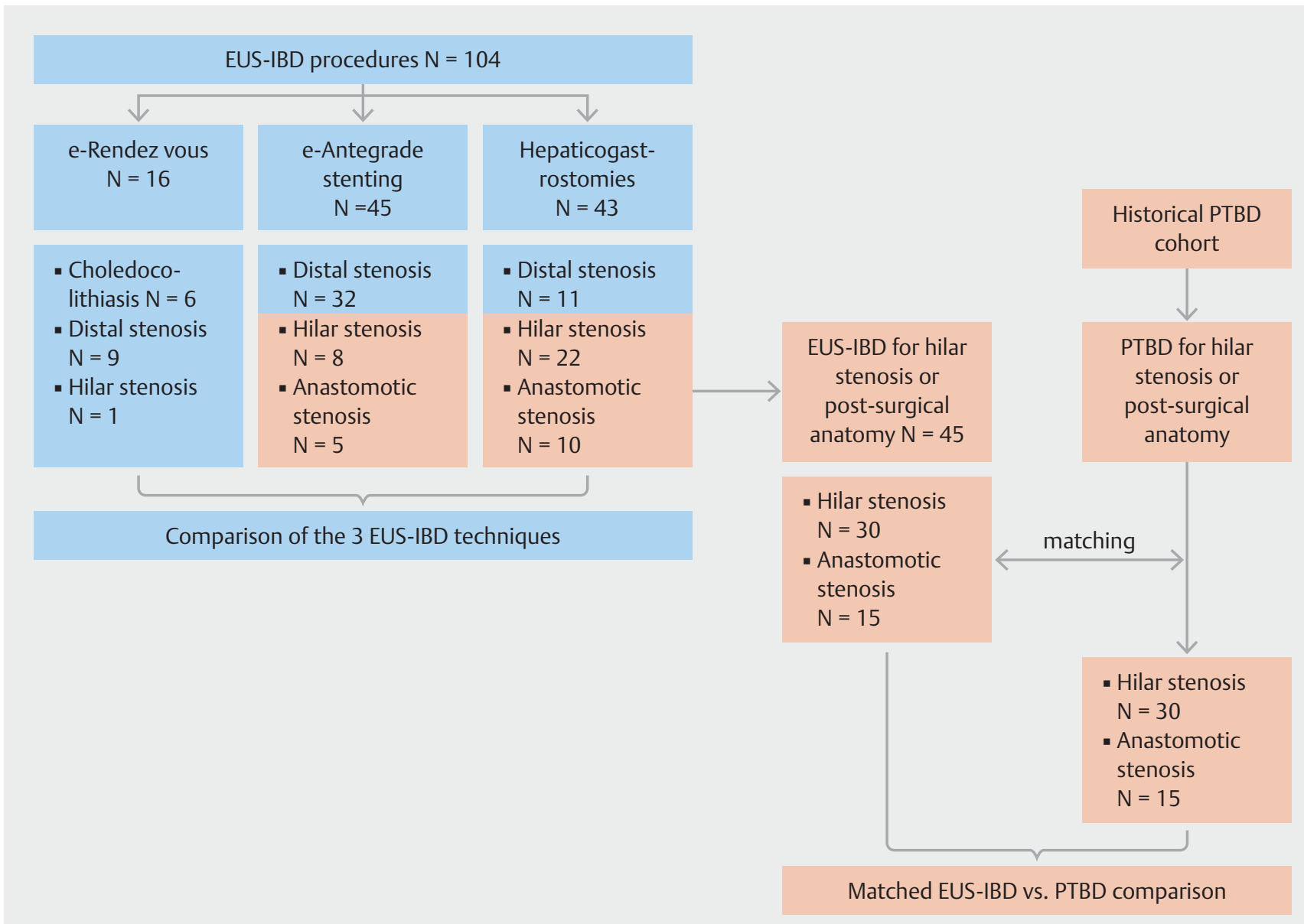

Fig. 1 Selection of patients. Between January 2012 and October 2019, 104 EUS-IBDs were performed. After transgastric intrahepatic access, e-RV was performed in 16 patients (15.4\%), e-AS in 43 (43.3\%) and e-HG in 45 (41.3\%). Outcomes of these three techniques were compared. We then identified EUS-IBDs performed for hilar/intrahepatic stenosis or in the setting of postsurgical anatomy. These procedures were matched to one PTBD case from an historical cohort of PTBDs executed in the same time frame, using the criteria described in the text. Finally, outcomes of 45 EUS-IBDs and 45 PTBD were compared. 
ble for inclusion for the primary aim of the study, independently from indication.

For the secondary aim, the subgroup of patients undergoing EUS-IBD for a hilar/intrahepatic stenosis or in the setting of postsurgical anatomy was separately identified. A historical cohort of PTBDs executed in the same time frame and for the same indications was used for a matched cohort comparison of EUS-IBD vs. PTBD. - Fig. 1 provides details about patient selection. For every patient in each cohort, information on the same set of variables was extracted (Appendix $\mathbf{1}$ ).

Eventual follow-up outside our Institution was ascertained through patient electronic medical files, linked to peripheral referring hospitals within our network. Continuous variables are presented as median (interquartile range [IQR]) and categorical variables as rates (proportions). Outcomes are reported as incidence (95\% confidence interval $[\mathrm{Cl}]$ ).
Each patient gave explicit consent to all procedures. The local Institutional Review Board approved the protocol of this study (Identifier $=$ S63970).

\section{Definitions}

EUS-IBD is defined as any interventional procedure involving a EUS-guided transgastric puncture of an intrahepatic bile duct of the left liver lobe.

We defined "disconnected ductal system" at cholangiography as the absence of a functional communication between the left and right hepatic ducts due to a hilar/intrahepatic stenosis.

EUS-IBD procedures were categorized as rendez-vous (e-RV), antegrade stenting (e-AS), and hepatico-gastrostomies (e-HG) while PTBD procedures as percutaneous antegrade stenting ( $\mathrm{p}$ $A S)$, external/internal drainage ( $p$-EID), and external drainage (p-ED). > Fig. 2 and Appendix 2 list complete definitions.
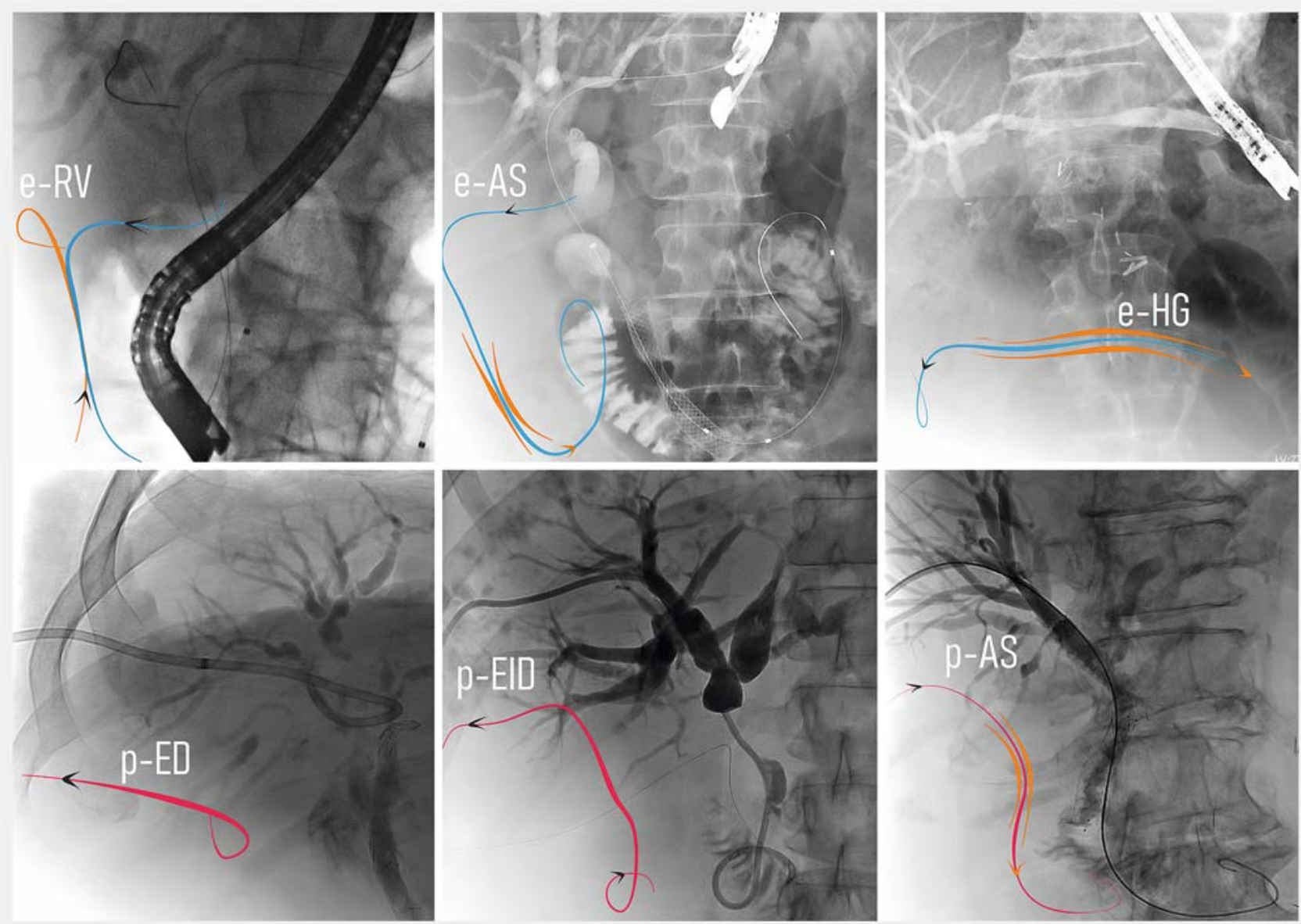

- Fig. 2 Endoscopic and percutaneous procedures described in this paper. Top: procedures following EUS-guided intrahepatic access. a EUSguided rendez-vous (e-RV) when EUS-IBD was used to allow antegrade transpapillary placement of a guidewire used for final retrograde therapeutic procedure (cannulation over or next to the guidewire). b EUS-guided antegrade stenting (e-AS) when a metal stent was advanced transgastric and transhepatic over a guidewire and finally placed bypassing a stenosis. c EUS-guided hepatico-gastrostomies (e-HG) when the drainage was guaranteed through the placement of a self-expanding metal stent (SEMS) between the left intrahepatic duct and the stomach. Bottom: Percutaneous procedures. $d$ Percutaneous external drainage ( $p-E D)$ when the stenosis could not be passed and drainage was obtained through a transhepatic externally-placed catheter connected to a drainage bag. e Percutaneous external/internal drainage ( $p$-EID) when a drainage was placed with an external trans-cutaneous tip and an internal transpapillary tip in the duodenum. $\mathbf{f}$ Percutaneous antegrade stenting (p-AS) when a metal stent was advanced transhepatic and finally placed bypassing a stenosis. 
For e-HGs, we defined as "purpose-specific stent" a partiallycovered self-expanding metal stent (SEMS) (Giobor, Taewoong Medical Inc., Gimpo, Korea) with an uncovered portion for intrahepatic placement and a covered portion crossing the liver parenchyma and gastric wall to end in the gastric lumen with the purpose of avoiding a bile leak or pneumoperitoneum. Previously used approaches included fully-covered SEMS (FCSEMS), partially covered SEMS (PCSEMS) or overlapping (uncovered + FC) SEMS.

We defined "technical success" as a successful intrahepatic access with the possibility to perform a subsequent therapeutic intervention (stone-extraction, balloon-dilation or biliary stenting).

We defined "clinical success" as laboratory or clinical improvement as detailed in Appendix 3.

American Society for Gastrointestinal Endoscopy (ASGE) lexicon [9] criteria were used to stratify adverse events (AEs) as mild/moderate, severe or fatal. Post-procedural pain conservatively treated without additional medical work-up was not considered among AEs but was systematically recorded.

We defined "dysfunction" as a new occurrence of cholestasis due to stent obstruction or dislocation after a clinically successful procedure. Time to dysfunction was registered. We defined as "reinterventions" any planned or unplanned procedure due to failure, recurrence or revision (including internalization of an ED).

"Procedure duration" was defined as the interval in minutes between first and last cholangiography image.

\section{Intervention: EUS-guided intrahepatic access}

All procedures were performed by (or under direct supervision of) three senior endoscopists highly experienced in interventional endosonography (SvdM, WL, HvM). A linear echo-endoscope was positioned in the proximal stomach. Dilated intrahepatic ducts were identified with the help of color Doppler. When a dilated segment 2/3 duct was identified, a $19 \mathrm{G}$ needle (EchoTip Ultra, Cook Medical or Expect, Boston Scientific) was advanced under ultrasonographic control (> Fig.3). The position of the needle tip in the duct was confirmed through bile aspiration and subsequent contrast injection. A 0.025-inch guidewire (Visiglide, Olympus) was inserted through the needle. When a stable position of the guidewire was obtained, the needle was exchanged for a 6 Fr cystotome (ENDO-FLEX GmbH, Voerde, Germany), used with cutting current under standard papillotomy settings on an ERBE electrosurgical unit (ERBE, Tübingen, Germany). Additional description of RVs, ASs or HGs is available in Appendix 2.

\section{Matching}

EUS-IBDs performed for hilar/intrahepatic stenosis or in the setting of postsurgical anatomy were identified and matched to one PTBD case ( $\mathbf{F i g . 1}$ ). Variables perceived to have an influence on outcomes were used for matching, after scrutinizing previous literature $[10,11]$. To obtain homogeneous pre-procedural probability of technical/clinical failure and subsequent dysfunction/recurrence an exact matching was obtained for: malignant vs benign indication; level of the stenosis hilar/intra-
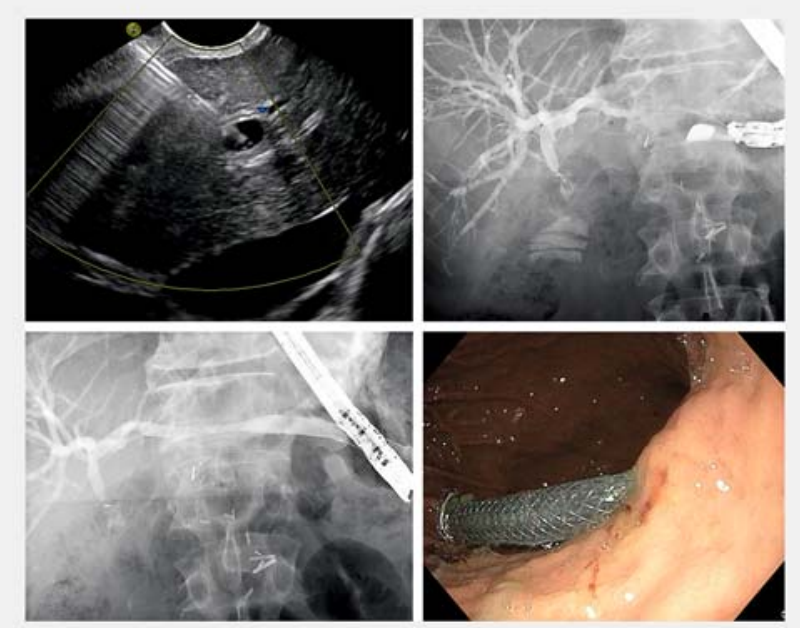

- Fig. 3 Hepaticogastrostomy. a Transgastric EUS-guided puncture of a dilated duct of left liver lobe. $\mathbf{b}$ Contrast injection and guidewire cannulation of the biliary tree. $\mathbf{c}$ After tract consolidation through cystotome, placement of a partially-covered SEMS with an uncovered portion for intrahepatic placement and a covered portion crossing the liver parenchyma and ending in the gastric lumen. d Endoscopic appearance of the covered part of the SEMS inside the gastric lumen.

hepatic vs. anastomotic; "disconnected ductal system" yes vs. no. A maximal difference of \pm 5 years was accepted for patients' age; differences in other variables not used for matching were explored and eventually discussed.

\section{Statistical analyses}

$\mathrm{X}$-squared test was used for comparing categorical variables, and differences in efficacy and safety shown as relative risk (RR) and $95 \% \mathrm{Cl}$. Mann-Whitney-U, Kruskal-Wallis and Wilcoxon signed rank tests were used for comparing continuous variables as appropriate. Kaplan-Meier curves were used for stent dysfunction-free survival and overall post-procedural survival, with log-rank test for comparisons. MedCalc Statistical Software (Ostend, Belgium) was used for matching and statistical analysis. $P<0.05$ was considered statistically significant.

\section{Results}

\section{EUS-guided intrahepatic access (EUS-IBD)}

104 EUS-IBDs were performed between January-2012 and October-2019 (\Table 1). Median age was $67(61-77)$ and $53.8 \%$ of patients were male.

The procedure indication was malignant stenosis in $87.7 \%$ of cases, benign stenosis in $10.6 \%$, and choledocholithiasis in $5.8 \%$. Stenosis was distal in $50 \%$, hilar/intrahepatic in $29.8 \%$, and anastomotic in $14.4 \%$ of cases. Of the procedures, $77.9 \%$ were executed after failed ERCP, while $22.1 \%$ were primary procedures due to surgery impeding access to the papillary area. 
- Table 1 Characteristics of included patients undergoing EUS-IBD.

\begin{tabular}{|c|c|}
\hline Variable & Total $\mathrm{N}=104$ \\
\hline Age, median [IQR] & $67[61-77]$ \\
\hline Male, n (\%) & $56(53.8 \%)$ \\
\hline \multicolumn{2}{|l|}{ Indication } \\
\hline - Malignancy, n (\%) & $87(83.7 \%)$ \\
\hline - Pancreatic cancer & 45 \\
\hline - Cholangiocarcinoma & 15 \\
\hline - Metastasis & 22 \\
\hline - Ampulloma/duodenal carcinoma & 5 \\
\hline - Benign disease, n (\%) & $17(16.3 \%)$ \\
\hline - Benign stricture & 11 \\
\hline - Anastomotic & 5 \\
\hline - Acute or chronic pancreatitis & 6 \\
\hline - Choledocholithiasis & 6 \\
\hline \multicolumn{2}{|l|}{ Level of stenosis, n (\%) } \\
\hline - Distal & $52(50 \%)$ \\
\hline - Hilar & $30(28.8 \%)$ \\
\hline - Anastomotic & $15(14.4 \%)$ \\
\hline - Intrahepatic & $1(1 \%)$ \\
\hline - Absent (choledocholithiasis) & $6(5.8 \%)$ \\
\hline Disconnected left biliary system, $\mathrm{n}(\%)$ & $27(26 \%)$ \\
\hline \multicolumn{2}{|l|}{ Reasons for transgastric approach } \\
\hline - Failed ERCP, n (\%) & $81(77.9 \%)$ \\
\hline $\begin{array}{l}\text { - Papillary region inaccessible (stenosis/infil- } \\
\text { tration) }\end{array}$ & 35 \\
\hline - Failed biliary cannulation & 45 \\
\hline - Stenosis not manageable by ERCP & 1 \\
\hline - Surgery impeding retrograde approach, n (\%) & $23(22.1 \%)$ \\
\hline - Whipple resection & 14 \\
\hline $\begin{array}{l}\text { - Hepatico-enterostomy after biliary/liver re- } \\
\text { sections }\end{array}$ & 5 \\
\hline - Distal gastrectomy & 3 \\
\hline $\begin{array}{l}\text { - Palliative gastro-enterostomy and hepatico- } \\
\text { enterostomy }\end{array}$ & 1 \\
\hline \multicolumn{2}{|l|}{ Procedure } \\
\hline $\begin{array}{l}\text { - EUS-guided transgastric ERCP rendez-vous } \\
\text { (e-RV) }\end{array}$ & $16(15.4 \%)$ \\
\hline $\begin{array}{l}\text { - EUS-guided transgastric antegrade biliary } \\
\text { stenting (e-AS) }\end{array}$ & $43(43.3 \%)$ \\
\hline - EUS-guided hepaticogastrostomy (e-HG) & $45(41.3 \%)$ \\
\hline
\end{tabular}

- Table 1 (Continuation)

\begin{tabular}{|l|c|}
\hline Variable & Total N=104 \\
\hline - Presence of ascites, n (\%) & $18 / 92(19.5 \%)$ \\
\hline - Presence of liver metastases, n (\%) & $23 / 91(25.3 \%)$ \\
\hline $\begin{array}{l}\text { EUS-IBD, endoscopic ultrasound-guided intrahepatic biliary drainage; IQR, } \\
\text { interquartile range; EUS, endoscopic ultrasound; ERCP, endoscopic retro- } \\
\text { grade cholangiopancreatography }\end{array}$ \\
\hline
\end{tabular}

\section{Procedures}

After transgastric intrahepatic access, e-RV was executed in 16 (15.4\%) patients, e-AS in 43 (43.3\%) and e-HG in 45 (41.3\%).

The procedure was performed despite the presence of ascites in 18 of 92 (19.6\%) and liver metastasis in 23 of 91 (25.3\%).

Successful intrahepatic access, cholangiogram, and guidewire cannulation was obtained in 93 of 104 patients (89.4\%) (Cl 81.8-94.6). However, in six cases the guidewire could not be advanced through the papilla into the duodenum and, due to concerns about possible subsequent surgical option, another way of drainage [e-CD] was preferred, lowering the per-protocol technical success to $87 / 104$ cases (83.7\% [Cl 75.2-90.2]).

Median procedure length was 35 minutes [24-56]. The clinical success rate was $96.2 \%(\mathrm{Cl} 89.8 \%-99.1 \%)$ among patients with available follow-up (79/87).

\section{Laboratory evaluation}

Among patients with biliary stenosis and available biochemistry, median pre-procedural and post-procedural bilirubin were $7(3.5-13.8)$ and $2.0(1.1-4.5) \mathrm{mg} / \mathrm{dL}(P<0.0001)$, with a reduction of $64.5 \%$ (42.2\% to $78 \%$ ) in 7 days (5-13) (Table S1).

Among all EUS-IBDs with available biochemistry, a Day-1 post-procedure increase of C-reactive protein was noticed in $76.2 \%$ (Cl 63.8-86.04), with increasing rates in patients with no symptoms, post-procedural pain, or AEs, $\mathrm{p}$-for-trend= 0.02). Analysis of serial measurements showed persistent Day7 elevation only among patients with AEs, while a significant reduction between Days 1 and 7 among the rest (TableS1).

\section{Radiological evaluation}

Post-procedural imaging was performed in 16 cases $(43.7 \%$ asymptomatic); Table S1. Results were negative for any finding in five plain $\mathrm{x}$-rays, showed subdiaphragmatic free air in two (12.5\%, all conservatively managed) and new or worsened pleural effusion or lung basal dys-/hypo-ventilation in nine (56.3\%).

\section{Adverse events}

Of the patients, $58.3 \%(\mathrm{Cl} 48.2-68)$ were asymptomatic after the procedure; 19 (18.4\%) (Cl 11.5-27.3) experienced mild abdominal pain not requiring any additional investigations; 24 (23.3\%) (Cl 15.6-32.7) experienced an adverse event (AE): severity was graded as mild/moderate, severe or fatal in 20 , three and one cases, respectively, with an overall incidence of 19.4\% (Cl 12.3-28.4), 2.9\% (Cl 0.6-8.3) and 0.9\% (Cl $0.02-$ 
5.2), respectively. The fatal case was a fragile patient who experienced overt bleeding suspected at computed tomography (CT) to originate from the hepaticogastrostomy access. $>$ Table 2 provides a complete list of AEs.

Median post-procedural hospital stay was $4.5(1-9)$ days in case of no AEs, 7 (3.5-8) days in case of mild post-procedural pain (post-hoc comparison not significant) and 10.5 (6-16.5) days in case of AEs $(P=0.005)$.

\section{Dysfunction}

After an initial clinical success, among 71 patients with a stenosis and available clinical follow-up, stent dysfunction occurred in $15.5 \%(\mathrm{Cl} 8-26.04)$ of patients (1 migration, 10 obstructions) after a median of 96 days (51.5 - 167). Proportion of dysfunction-free survival was estimated to be $98.1 \%$ (Cl 94.5100), 87\% (Cl 76.1-97.8) and 68.5\% (Cl 49.7-87.3) at 1, 3, and 6 months on Kaplan-Meyer curves ( $>$ Table 2). The median number of reinterventions for the whole group was $0(0-0)$.

\section{Comparison of EUS-IBD procedures}

Benign diseases (choledocholithiasis or benign stenosis) represented $50 \%$ of indications in the e-RV group, while $91.1 \%$ of eASs and $88.4 \%$ of e-HGs were done for malignant disease $(P=$ $0.0004)$. A stenosis was more frequently absent among e-RVs, while it was more frequently hilar/anastomotic among e-HGs (51.2\% vs. $17.8 \%$ of e-ASs and $6.2 \%$ of e-RVs; $P<0.0001)$. A higher proportion of patients with postsurgical anatomy was treated through e-HG $(P=0.01)$; $\triangleright$ Table 3 .

Technical success was lower among e-RVs $(P=0.04)$, but one-third of failures in this group were represented by successful biliary cannulation but subsequent impossibility to pass a guidewire transpapillary, with a choledochoduodenostomy preferred over hepaticogastrostomy.

No difference in per-protocol clinical success was identified.

A smaller decrease in bilirubin was seen among e-HGs $(\geq 50 \%$ decrease in $51.6 \%(\mathrm{Cl} 33.1-69.8)$ vs. $66.7 \%(\mathrm{Cl} 9.4-99.2)$ and $89.7 \%(\mathrm{Cl} 72.7-97.8$ ) of e-RVs and e-ASs respectively; $P=$ 0.01 ), which might be related to the higher rate of "disconnected" left biliary system among this subgroup (53.5\% vs. 6.2 and $6.7 \%$ of e-RVs and e-ASs, $P<0.0001$ ).

No significant difference in the rate of 1) post-procedural symptomatic patients, 2) AEs or severe AEs was noted.

$A E s$ was noted in the rate of severe AEs (no severe AE was detected among e-RVs). The incidence of stent dysfunction and time to dysfunction were not different ( $\vee$ Table 3 ).

\section{Subgroup analysis of hepatico-gastrostomies}

Among hepatico-gastrostomies ( $\mathrm{HGs})(\mathrm{N}=43)$, the rate of technical success was $88.4 \%(\mathrm{Cl} 75-96.1)$. HG was created through a purpose-specific stent in 16 cases (37.7\%), while other approaches were used in the remaining cases (Table S2).

A significantly shorter procedural time was observed with the use of a purpose-specific stent (median 25 vs. 48 minutes, $P=0.004)$ together with a trend toward less stent dysfunction (6.7\% [Cl 0.2-32] vs. $30 \%(\mathrm{Cl} 11.9-54.3), P=0.09 ; \mathrm{RR}=0.2$ [Cl $0.03-1.7])$.

\section{Subgroup comparison of EUS-guided vs. percutaneous BD in patients with proximal stenoses or postsurgical anatomy}

Of 104 EUS-IBDs, 45 procedures were performed for a hilar/intrahepatic stenosis or in a patient with postsurgical anatomy impeding access to papillary region.

Those 45 patients were matched 1:1 with an historical cohort of PTBDs using the aforementioned criteria. Of EUS-IBD patients, $38.6 \%$ had already received a previous biliary drainage versus $17.8 \%$ of PTBD, $P=0.03$. No other differences were noticed in non-matched variables.

Among PTBDs, p-ED was performed in eight patients (18\%), p-EID in 21 patients (47.7\%) and p-AS in 15 (34\%). However, an external coaxial drain was left inside the metal stent in five cases, so that the number of patients with primary placement of an external catheter was 34 (75.6\%). Subsequent internalization of an external drainage was possible in 18 (52.9\%, in three by means of e-HG), while in 11 and five cases, respectively internalization was impossible or only temporary. Comparisons between EUS-IBDs and PTBD groups are reported in $>$ Table 4.

Technical success rates were equally high, but clinical success rates were significantly higher in the EUS group $(97.4 \%$ [Cl 86.5-99.9] vs 79.5\% [Cl 65-90.4], $P=0.01 ; \quad R R=1.23$ [1.05-1.44]).

A significantly higher proportion of patients in the EUS-IBD group did not experience any post-procedural clinical event or symptom $(64.4 \%[\mathrm{Cl} 48.7-78.1]$ vs $31.1 \%$ [Cl 18.2-46.6], $P=$ $0.004 ; \mathrm{RR}=2.07$ [1.27-3.37]), while $44.4 \%(\mathrm{Cl} 29.6-60)$ of PTBDs experienced post-procedural abdominal pain (vs $17.8 \%$ [CI 8-32.1] of EUS-IBDs, $P=0.004 ; \mathrm{RR}=2.5$ [1.23-5.08]), even if the rate of overall and severe AEs was not different.

Median post-procedural hospitalization was 7.5 days in EUSIBD and 11.5 in PTBD group $(P=0.01)$.

There was a significantly higher rate of stent dysfunction in the PTBD group (42.9\% [Cl 26.4-60.7] vs.15.8\% [Cl 6-31.3], $P=0.01, R R=2.71[1.19-6.21])$ and a higher need for post-procedural reinterventions (median $1[0.25-3]$ vs. $0[0-0], P<$ $0.0001)$.

\section{Discussion}

This large, retrospective, single-center experience shows that EUS-guided intrahepatic biliary access has high rates of technical and clinical success with an acceptable safety profile. Hepaticogastrostomy showed equivalent outcomes when compared to rendez-vous and antegrade stenting with regards to success and AEs rate. In addition, the success of hepaticogastrostomy was slightly superior when purpose-specific stents were used. For hilar/intrahepatic strictures and patients with surgically-altered anatomy, EUS-guided approach seems safer, more effective and less prone to stent dysfunction when compared to PTBDs with the same indications. These results are of great clinical importance due to the paucity of comparative data, as well as the morbidity associated with PTBD in a population already stricken by reduced quality of life. 
- Table 2 General Outcomes of EUS-IBDs.

\begin{tabular}{|c|c|}
\hline Variable & Total $\mathrm{N}=104$ \\
\hline \multicolumn{2}{|l|}{ Technical success } \\
\hline - Technical failures, n (\%) & $11(10.6 \%)$ \\
\hline - Biliary tree never opacified & 4 \\
\hline - Impossible guidewire cannulation & 2 \\
\hline - Guidewire dislocation after access & 2 \\
\hline - Impossible to place a HG & 2 \\
\hline - Scope-related technical issues & 1 \\
\hline $\begin{array}{l}\text { - Successful intrahepatic access and guidewire } \\
\text { cannulation, } \mathrm{n}(\%)\end{array}$ & $93 / 104(89.4 \%)$ \\
\hline - Multiple biliary punctures required & 5 \\
\hline $\begin{array}{l}\text { - Hepaticogastrostomy stent misplacement, } \\
\text { saved intraprocedurally with coaxial FC-SEMS }\end{array}$ & 5 \\
\hline $\begin{array}{l}\text { - Impossibility to place the guidewire transpa- } \\
\text { pillary } \rightarrow \text { extrahepatic EUS-guided drainage } \\
\text { preferred over HG }\end{array}$ & 6 \\
\hline - Complete technical success, n (\%) & $87 / 104(83.7 \%)$ \\
\hline - Median procedural length [IQR], minutes & $35[24-56]$ \\
\hline \multicolumn{2}{|l|}{ Clinical success } \\
\hline - Complete procedures, n (\%) & $N=87$ \\
\hline - Treatment of choledocholithiasis & $4 / 4$ \\
\hline - Biliary stenosis ${ }^{1}$ & $72 / 75^{1}$ \\
\hline - Overall clinical success, n (\%) ${ }^{1}$ & $76 / 79(96.2 \%)$ \\
\hline \multicolumn{2}{|l|}{ Adverse events } \\
\hline - Available follow-up & $N=103$ \\
\hline - No clinical event, n (\%) & $60(58.3 \%)$ \\
\hline - Mild self-limiting post-procedural pain, n (\%) & $19(18.4 \%)$ \\
\hline - Overall adverse events rate, $\mathrm{n}(\%)$ & $24(23.3 \%)$ \\
\hline \multicolumn{2}{|l|}{ Timing, $\mathrm{n}$} \\
\hline - Intraprocedural & 3 \\
\hline - Same-day post-procedural & 11 \\
\hline - Early (<7 days) & 10 \\
\hline - Late & 0 \\
\hline \multicolumn{2}{|l|}{ Type, $n$} \\
\hline - Perforation (2/2 surgical management) & 2 \\
\hline - Bleeding & 3 \\
\hline - Mild hemobilia (no management required) & $1 / 3$ \\
\hline $\begin{array}{l}\text { - Hemorrhagic shock (endovascular treat- } \\
\text { ment) }{ }^{2}\end{array}$ & $1 / 3$ \\
\hline - Hemoperitoneum (treated conservatively) & $1 / 3$ \\
\hline - Bile leak + peritonitis (treated conservatively) & 1 \\
\hline - Cholangitis & 9 \\
\hline - Bacteremia & 3 \\
\hline
\end{tabular}

- Table 2 (Continuation)

Variable

Total $\mathbf{N}=104$

\begin{tabular}{l|l}
\hline - Acute pancreatitis & 4 \\
\hline - Severe abdominal pain & 2 \\
\hline Severity (ASGE lexicon), $\mathrm{n}(\%)$ & \\
\hline - Mild/moderate & $20 / 103(19.4 \%)$ \\
\hline - Severe & $3 / 103(2.9 \%)$ \\
\hline - Fatal & $1 / 103(0.9 \%)$ \\
\hline
\end{tabular}

Median post-procedural length of hospital stay [IQR], days

- All patients with available follow-up $(\mathrm{N}=79) \quad 7[3-10]$

- No clinical event $4.5[1-9]^{3}$

- Mild post-procedural pain $7[3.5-8]^{3}$

- Patients with adverse events $10.5[6-16.5]^{3}$

Follow-up

Stent dysfunction ${ }^{4}$

- Median post-procedural FU $(\mathrm{N}=71)$

[16.3-135.8]

- No stent dysfunction, $\mathrm{n}(\%)$

$60 / 71(84.5 \%)$

- Stent dysfunction, $\mathrm{n}(\%)$

$11 / 71(15.5 \%)$

- Stent migration

1

- Stent obstruction (clots/ingrowth) 10

- Time to dysfunction [IQR], days $96[51.5-167]$

Rescue procedures

- None 2

- ERCP 4

- New HG 3 (1 using the

same fistula)

- SEMS-in-SEMS of the HG $\quad 1$

- Plastic stenting of the HG 1

- $1,3,6,12$ months probability of no dysfunc- $\quad 98.1 \%, 87 \%$, tion $^{5}$

$68.5 \%, 61.7 \%$

Median number of reinterventions, [IQR] $\quad 0[0-0]$

EUS-IBD, endoscopic ultrasound-guided intrahepatic biliary drainage; HG, hepaticogastrostomy; FCSEM, fully-covered self-expanding metal stent; EUS, endoscopic ultrasound; IQR, interquartile range; FU, follow-up.

${ }^{1}$ Excluding lost-to-follow-up $(\mathrm{N}=8)$

${ }^{2}$ Fatal event

$3 P=0.005$ of Kruskal-Wallis test for 3 -groups comparison, post-hoc analysis showing significantly different length only between patients with adverse events versus each other subgroup.

${ }^{4}$ Per-protocol; among patients with biliary stenosis and successful stent placement

${ }^{5}$ Kaplan-Meier curves 
- Table 3 Comparison of the three EUS-IBD techniques.

\begin{tabular}{|c|c|c|c|c|}
\hline Variable & $e-R V(N=16)$ & e-AS $(N=45)$ & e-HG $(N=43)$ & $P$ value \\
\hline \multicolumn{5}{|l|}{ Clinical indication } \\
\hline Proportion of malignant indication, $\mathrm{n}(\%)$ & $8(50 \%)$ & $41(91.1 \%)$ & $38(88.4 \%)$ & $0.0004^{1}$ \\
\hline Level of the stenosis, $\mathrm{n}(\%)$ & & & & $<0.0001^{1}$ \\
\hline None (choledocholithiasis) & $6(37.5 \%)$ & 0 & 0 & \\
\hline Distal & $9(56.2 \%)$ & $32(71.1 \%)$ & $11(25.6 \%)$ & \\
\hline Hilar/intrahepatic & $1(6.2 \%)$ & $8(17.8 \%)$ & $22(51.2 \%)$ & \\
\hline Anastomotic (after surgical hepatico-enterostomy) & 0 & $5(11.1 \%)$ & $10(23.3 \%)$ & \\
\hline Reason for the transgastric approach, $\mathrm{n}(\%)$ & & & & $0.01^{1}$ \\
\hline Surgery impeding access to papillary region & 0 & $9(20 \%)$ & $14(32.6 \%)$ & \\
\hline Papillary region inaccessible for stenosis/infiltration & $4(25 \%)$ & $22(48.9 \%)$ & $9(20.9 \%)$ & \\
\hline Failed ERCP cannulation & $12(75 \%)$ & $14(31.1 \%)$ & $19(44.2 \%)$ & \\
\hline Successful ERCP but unnegotiable stenosis & 0 & 0 & $1(2.3 \%)$ & \\
\hline "Disconnected ductal system", n (\%) & $1(6.2 \%)$ & $3(6.7 \%)$ & $23(53.5 \%)$ & $<0.0001^{1}$ \\
\hline \multicolumn{5}{|l|}{ Efficacy } \\
\hline Technical success, n (\%) & & & & $0.04^{1}$ \\
\hline Successful access and complete treatment & $10(62.5 \%)$ & $39(86.7 \%)$ & $38(88.4 \%)$ & \\
\hline Successful biliary cannulation but uncomplete procedure & $2(12.5 \%)$ & $4(8.9 \%)$ & 0 & \\
\hline Technical failure, n (\%) & $4(25 \%)$ & $2(4.4 \%)$ & $5(11.6 \%)$ & \\
\hline Clinical success, n (\%) & $8 / 8(100 \%)$ & $32 / 33(97 \%)$ & $36 / 38(94.7 \%)$ & 0.74 \\
\hline Bilirubin decrease $\geq 25 \%, \mathrm{n}(\%)^{2}$ & $2 / 3(66.7 \%)$ & $28 / 29(96.6 \%)$ & $27 / 31(87.1 \%)$ & 0.16 \\
\hline Bilirubin decrease $\geq 50 \%, n(\%)^{2}$ & $2 / 3(66.7 \%)$ & $26 / 29(89.7 \%)$ & $16 / 31(51.6 \%)$ & $0.01^{1}$ \\
\hline Stent dysfunction, $\mathrm{n}(\%)^{3}$ & $0 / 4(0 \%)$ & $4 / 32(12.5 \%)$ & $7 / 35(20 \%)$ & 0.47 \\
\hline Median time to dysfunction [IQR], days ${ }^{3}$ & - & $101[49.5-147.5]$ & $96[51.5-182]$ & $0.71^{4}$ \\
\hline \multicolumn{5}{|l|}{ Safety } \\
\hline Acute increase of inflammatory markers, $\mathrm{n}(\%)^{5}$ & $4 / 6(66.7 \%)$ & $23 / 26(88.5 \%)$ & $21 / 31(67.7 \%)$ & 0.16 \\
\hline Adverse events, n (\%) & & & & 0.83 \\
\hline Mild abdominal pain & $3 / 15(20 \%)$ & $7 / 45(15.6 \%)$ & $9 / 43(20.9 \%)$ & \\
\hline Any adverse events & $2 / 15(13.3 \%)$ & $11 / 45(24.4 \%)$ & $11 / 43(25.6 \%)$ & \\
\hline Severe adverse events & $0 / 2(0 \%)$ & $2 / 11(18.2 \%)$ & $2 / 11(18.2 \%)$ & 0.8 \\
\hline Median post-procedural survival [IQR], days & $76[59.8-428.8]$ & $61[39-185]$ & $50[24.3-156]$ & $0.24^{6}$ \\
\hline \multicolumn{5}{|c|}{$\begin{array}{l}\text { EUS-IBD, endoscopic ultrasound-guided intrahepatic biliary drainage; IQR, interquartile range; EUS, endoscopic ultrasound; ERCP, endoscopic retrograde } \\
\text { giopancreatography } \\
1 \text { Statistically significant } \\
2 \text { Per-protocol; among patients with pre-procedural bilirubin elevation } \\
{ }^{3} \text { Per-protocol; among patients with biliary stenosis and successful stent placement } \\
{ }^{4} \mathrm{No} \text { different probability of dysfunction-free survival at log-rank test }(\mathrm{P}=0.1908) \\
{ }^{5} \mathrm{Among} \text { patients with technical success } \\
{ }^{6} \mathrm{Higher} \text { probability of survival among patients undergoing e-RV versus e-AG }(\mathrm{HR}=2[1.1-3.6]) \text { and e-HG (HR=2.1 [1.1-3.9]); log-rank test }(P=0.1186)\end{array}$} \\
\hline
\end{tabular}


Table4 EUS-IBD versus percutaneous biliary drainage for patients with proximal stenosis or post-surgical anatomy.

\begin{tabular}{|c|c|c|c|}
\hline Variable & EUS-IBD $(N=45)$ & PTBD ( $N=45)$ & $P$ value \\
\hline \multicolumn{4}{|l|}{ Matched variables } \\
\hline Proportion of malignant indication, $\mathrm{n}$ (\%) & $40(88.9 \%)$ & $40(88.9 \%)$ & 1 \\
\hline Level of the stenosis, $\mathrm{n}(\%)$ & & & 1 \\
\hline Anastomotic & $15(33.3 \%)$ & $15(33.3 \%)$ & \\
\hline Hilar/Intrahepatic & $30(66.7 \%)$ & $30(66.7 \%)$ & \\
\hline “Disconnected ductal system”, n (\%) & $26(57.8 \%)$ & $26(57.8 \%)$ & 1 \\
\hline Age [IQR], years & $67[60.5-76]$ & $67[62.3-74.5]$ & 0.96 \\
\hline \multicolumn{4}{|l|}{ Other Variables } \\
\hline Male sex & $23(51.1 \%)$ & $30(66.7 \%)$ & 0.1 \\
\hline Previous failed ERCP & $18(40 \%)$ & $18(40 \%)$ & 1 \\
\hline Previous biliary drainage & $17(38.6 \%)$ & $8(17.8 \%)$ & $0.03^{1}$ \\
\hline Median bilirubin [IQR], mg/dl & $5.1[2.6-10.2]$ & $8.3[2.9-13.3]$ & 0.2 \\
\hline \multicolumn{4}{|l|}{ Efficacy } \\
\hline Technical success, n (\%) & $42(93.3 \%)$ & $44(97.8 \%)$ & 0.31 \\
\hline Available FU & $N=39$ & $N=44$ & \\
\hline Clinical success, n (\%) & $38(97.4 \%)$ & $35(79.5 \%)$ & $0.01^{1}$ \\
\hline Median bilirubin decrease [IQR], mg/dL ${ }^{2}$ & $2.6[1.2-5.2](N=33)$ & $4.2[0.9-7](N=39)$ & 0.64 \\
\hline Time to bilirubin decrease [IQR], days ${ }^{2}$ & $7[5-11]$ & $6[3-16]$ & 0.44 \\
\hline Bilirubin decrease $\geq 25 \%, n(\%)^{2}$ & $30 / 33(90.9 \%)$ & $31 / 39(79.5 \%)$ & 0.18 \\
\hline Bilirubin decrease $\geq 50 \%, n(\%)^{2}$ & $18 / 33(54.5 \%)$ & $20 / 39(51.3 \%)$ & 0.78 \\
\hline Procedural time [IQR], minutes & $35[24.8-60.3]$ & $45[28.5-69.5]$ & 0.23 \\
\hline Median hospital stay [IQR], days & $7.5[2-10](N=34)$ & $11.5[7-21.5](N=44)$ & $0.01^{1}$ \\
\hline Safety & & & $0.004^{1}$ \\
\hline No post-procedural event, $\mathrm{n}(\%)$ & $29(64.4 \%)$ & $14(31.1 \%)$ & \\
\hline Mild post-procedural pain, $n(\%)$ & $8(17.8 \%)$ & $20(44.4 \%)$ & \\
\hline Adverse events, n (\%) & $8(17.8 \%)$ & $11(24.4 \%)$ & \\
\hline Severe adverse events, n (\%) & $1(2.2 \%)$ & $0(0 \%)$ & 0.32 \\
\hline \multicolumn{4}{|l|}{ Follow-up } \\
\hline Stent dysfunction, $\mathrm{n}(\%)^{3}$ & $6 / 38(15.8 \%)$ & $15 / 35(42.9 \%)$ & $0.01^{1}$ \\
\hline Median time to dysfunction [IQR], days & $118[77-196]$ & $81[20-157]$ & $0.31^{4}$ \\
\hline Median number of reinterventions [IQR] & $0[0-0]$ & $1[0.25-3]$ & $<0.0001^{1}$ \\
\hline Median post-procedural survival $(95 \% \mathrm{Cl})^{5}$, days & $91(95 \% \mathrm{Cl} 50-168)$ & $119(95 \% \mathrm{Cl} 77-250)$ & $0.61^{5}$ \\
\hline $\begin{array}{l}\text { EUS-IBD, endoscopic ultrasound-guided intrahepatic } \\
\text { cholangiopancreatography. } \\
{ }^{1} \text { Statistically significant } \\
{ }^{2} \text { Per-protocol; among patients with available data an } \\
{ }^{3} \text { Per-protocol; among patients with clinical success a } \\
{ }^{4} \mathrm{~N} \text { differences in dysfunction-free survival at log-ran } \\
{ }^{5} \text { Based on the log-rank test at Kaplan-Meier statistics }\end{array}$ & $\begin{array}{l}\text { e; PTBD, percutaneous bilic } \\
\text { ral elevation } \\
\text { llow-up }\end{array}$ & ; IQR, interquartile range; $E$ & copic retrog \\
\hline
\end{tabular}


Although a recent review on EUS-guided BD summarized the cumulative technical success, clinical success, and AE rates of $95 \%, 92 \%$, and $23 \%$, respectively [3], literature describing intrahepatic drainage is typically composed of small retrospective series [12-15]. Larger series usually include miscellaneous procedures (e.g. both extrahepatic and intrahepatic routes of EUS-BD) $[8,16-18]$ or different indications, so that it becomes difficult to position this technique in management algorithms. Techniques are not standardized (e.g. with/without tract dilation, cystotome vs. needle-knife) and results lack distinction according to adopted devices (e.g. plastic vs. metal stents, type of SEMS) $[8,16]$. The only retrospective experience describing specifically designed half-covered SEMS, has no comparator [19].

We describe 104 consecutive EUS-IBD cases executed in a single center, with a standardized protocol. Our results are in line with previous literature; the slightly higher incidence of technical failures may be due to the evolution of expertise over time but also related to our rigid definition of failures on an intention-to-treat basis. Of note, in six of 17 "failures" biliary access, opacification of the biliary tree and guidewire cannulation were successful, but the guidewire could not be manipulated to transverse the distal stenosis to allow for antegrade stenting and alternative drainage using EUS-guided choledochoduodenostomy was preferred above hepaticogastrostomy. Among the remaining 11 failures, four were due to poorly dilated biliary tree, while five were related to manipulation of guidewires and accessories, advocating for a stricter selection of patients in terms of technical aspects and development of urgently needed single-step devices to overcome technical hazards/concerns. We were not able to analyze predictors of technical failures in our series, but previous studies suggested a dilation $\leq 5 \mathrm{~mm}$ of the punctured duct and a traversed hepatic parench$y m a \geq 3 \mathrm{~cm}$ as independent predictors of technical failure (without any role of choice of segment two vs. three for puncture or the presence of concomitant ascites) [20]. Ascites was detected in about $20 \%$ of our patients, but this did not prevent a stable position and good apposition of the gastric wall and the liver surface during the procedure; however, significant ascites should be considered a contraindication to EUS-guided intrahepatic transgastric approach, unless a pre-procedural drainage is performed.

AEs were present in $23.3 \%$ of our patients, with overall incidences of severe or fatal events of only $2.9 \%$ and $0.9 \%$ ( 1 case), respectively. Most events (18.4\%) consisted of self-limiting mild abdominal pain, not requiring any escalation of medical care. Upon radiological post-procedural surveillance, we noted a $12.5 \%$ rate of free subdiaphragmatic air, without any clinical consequences, to be considered an incidental finding after the transgastric approach. As for comparisons among different procedures following intrahepatic (IH) access, "pure" HGs, usually perceived technically more challenging among EUS-IBD procedures, demonstrated a higher technical success, an equally high clinical success, as well as comparable AEs and dysfunction rates compared to e-RV and e-AS. Moreover, HGs created with purpose-specific partially-covered stents were associated with reduced procedure duration (25 vs. 48 minutes) and a trend toward reduced stent dysfunction over time. However, even if some alternative approaches (e. g. two overlapping SEMS) are intuitively trickier and more time consuming, we cannot exclude a learning curve effect on the improved procedure duration.

The current literature is also limited with regard to the analysis of EUS-IBD versus comparators. Randomized clinical trials (RCTs) of EUS-guided BD versus ERCP [21] or PTBD [22,23] for distal malignant biliary obstruction (MBO) have included both extrahepatic and intrahepatic routes of drainage. This was also the case in two meta-analyses [24,25]. Extrahepatic and intrahepatic routes showed similar success in head-to-head RCTs [26] and pooled analyses [27]. However, sporadic reports of a safer profile $[7,8]$ and reduced complexity with electrocautery-enhanced lumen-opposing metal stent (LAMS) [4], actually support the extrahepatic route (e-CD) as a first-line rescue of failed ERCPs. However, e-CD is limited to distal stenoses, requires a significant common bile duct dilation (at least $10 \mathrm{~mm}$ ) and can be complicated by a duodenal infiltration [28], so that in an intention-to-treat scenario, feasibility is suboptimal and there is still room for complementary procedures.

Evidence becomes even more scant when dealing with proximal stenoses or patients with postsurgical anatomy, for which no possibility of extrahepatic drainage exists. Enteroscopy-assisted ERCP is theoretically an option in postsurgical anatomy, but reduced technical/clinical success, increased procedure duration and frequent AEs have been reported [2]. Therefore, clinical decisions for these patients typically involve a choice between EUS-BD and PTBD. The literature supports the superiority of EUS in terms of clinical efficacy and reduced AEs and reinterventions, but usually does not make distinctions between HGs and CDs $[22,29]$ and is focused on distal MBO, explicitly excluding hilar/intrahepatic stenosis [30,31]. Only initial experience has been published on EUS-IBD for hilar strictures $[32,33]$, so that no evidence exists on EUS-IBD vs. PTBD for patients for whom this decision is most relevant.

Therefore, we identified the subgroup of EUS-IBDs patients with proximal stenosis or postsurgical anatomy and compared them with an historical PTBD cohort, matched for clinical indication and biliary anatomy. This population, for which ERCP is precluded or has failed, represents a known clinical challenge, with an expected lower pre-procedure probability of clinical success, especially in the setting of "disconnected" left and right hepatic ducts. Despite this, we demonstrated that in this specific scenario, rates of clinical success were higher among EUS-IBDs. This is particularly valuable, considering the higher rate of previous $\mathrm{BD}$ in the endoscopic group, suggestive of a more advanced disease course. Overall and severe AEs were similar, but patients in the PTBD group experienced more frequent post-procedural pain and longer hospital stay. During follow-up, both stent dysfunction and overall need-for-reinterventions were higher in the PTBD group, although there was no difference in post-procedural survival.

Moreover, when the guidewire fails to cross the stricture, the alternative to EUS-IBD is a transgastric stent (HG), whereas for PTBD, the only alternative is external drainage, which is associated with increased discomfort and reduced quality of life. 
Of note, even if it is routine practice at our hospital to aim for internalization of every external PTBD, $47.1 \%$ of percutaneous catheters could not be removed. In these cases, the possibility of subsequent elective internalization through EUS-HG must be considered [34]

For all these reasons, our data support primary EUS-IBD over PTBD in cases of proximal stenosis or surgically-altered anatomy. Furthermore, rate of AEs of EUS-IBD is expected to decrease, hand-in-hand with increased expertise and the development of new devices (e.g. single-step stent introducers [35]). However, PTBD remains complementary to endoscopy, in cases of EUS failure or need to selectively drain the right biliary tree, even if proof-of-concept studies are starting to overcome this left-EUS / right-PTBD dichotomy [36].

In general, the ability with endoscopy to rapidly switch to another access or approach in the same session under the same sedation is an additional advantage over percutaneous access to personalize treatment according to biliary anatomy [37] ( Fig. 4).

This study has several limitatoins. First of all, the retrospective nature is theoretically prone to under-detection of events during follow-up. However, the fully computerized medical records used in our hospital and in many facilities in our network reduced this risk. Follow-up was not available for every patient, but the novelty of these procedures and the hub-spoke model of healthcare in our region significantly reduced the likelihood that AEs were managed outside (or without consultation with) our center. Procedures were executed in a tertiary referral center with high expertise on biliopancreatic endoscopy, therefore, reproducibility cannot be guaranteed outside this setting. This is also true for PTBD procedures, and the advantages of the EUS-guided approach in our matched comparison might be underestimated by the excellent performance and dexterity of our interventional radiologists, as demonstrated by the low rate of overall and severe AEs when compared with published literature [22, 31]. Finally, the number of included procedures is limited, and given the exploratory nature of the study, no correction for multiple testing was performed; these factors expose the study to the risk of both type 1 and type 2 error when performing comparisons, therefore, the results must be critically read and further tested through more studies that are fully powered.

Despite all these limitations, this is one of the largest published experiences with EUS-guided intrahepatic access for BD, one of the few using a standardized cystotome-guided dilationfree technique for tract creation, the only one to separately de-

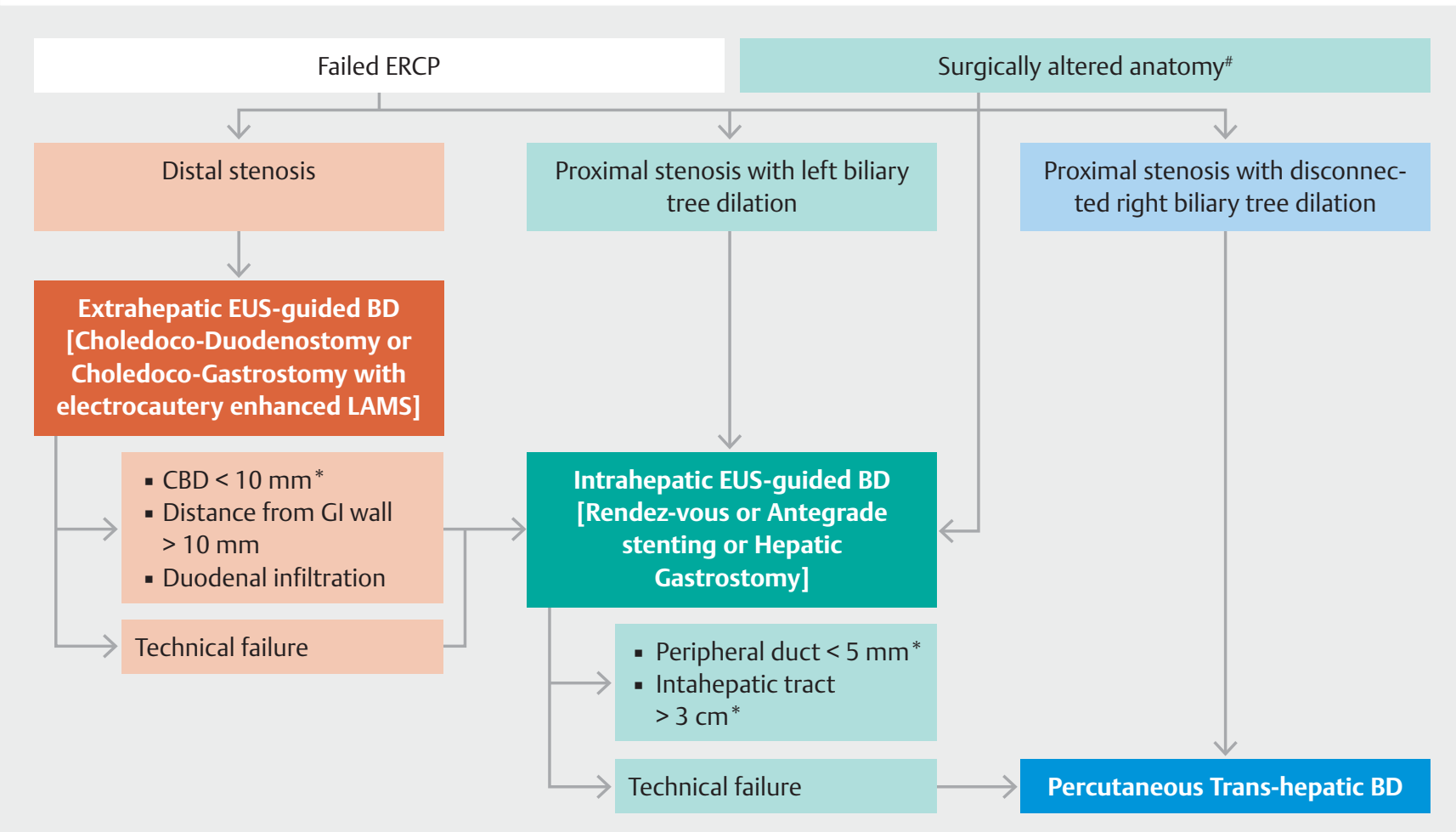

-Fig. 4 Proposed algorithm for the management of biliary obstruction. In case of postsurgical anatomy, when papillary region is not accessible, EUS-guided intrahepatic biliary drainage (EUS-IBD) may represent the first-line treatment modality. In case of biliary stenosis and failed ERCP: 1) when stenosis is distal and the common bile duct is significantly dilated we propose extrahepatic drainage through an electrocautery-enhanced LAMS as the first-line treatment; 2 ) when the stenosis is proximal and determines a dilation of the left biliary tree we propose EUS-IBD as the first-line treatment; and 3) when the stenosis determines an isolated dilation of the right biliary tree (or other modalities have failed) we propose percutaneous biliary drainage (PTBD).

${ }^{*}$ The cut-off included in the algorithm is taken from studies cited in the text, but may vary according to specific cases and local expertise.

" Post-surgical anatomy impeding access to papillary area (e. g. pancreaticoduodenectomy), with the exception of Roux-en-Y gastric bypass. 
scribe the three procedures following intrahepatic access, and the only one reporting outcomes of HGs executed with specifically designed stents compared with previous approaches. Finally, our comparison of EUS-IBD vs. PTBD, although limited by the small sample size, originally included only patients with proximal stricture or surgically-altered anatomy.

\section{Conclusion}

Based on our data and on previously published literature, we believe that the time is ripe to consider extrahepatic and intrahepatic EUS-guided accesses as different, though complementary, procedures with different initial indications. Therefore, we propose a new to-be-validated clinical algorithm ( Fig.4) based on the proposition that there is a specific place for EUSIBD in the multidisciplinary armamentarium of tertiary centers managing biliopancreatic diseases.

\section{Acknowledgements}

The authors acknowledge the expertise and commitment of our dedicated Interventional Gastroenterology nursing team as well as the fellows and gastroenterology trainees that help to care for our patients.

\section{Competing interests}

Dr. van der Merwe holds the Cook chair in Interventional endoscopy and holds consultancy agreements with Cook, Pentax and Olympus. Dr. Laleman co-chairs the Boston Scientific Chair in Therapeutic Biliopancreatic Endoscopy with Dr. van der Merwe and has consultancy agreements with Boston Scientific and Cook. Dr. Van Malenstein holds a consultancy agreement with Boston Scientific

\section{References}

[1] Enochsson L, Swahn F, Arnelo U et al. Nationwide, population-based data from 11,074 ERCP procedures from the Swedish Registry for Gallstone Surgery and ERCP. Gastrointest Endosc 2010: 72 Epub ahead of print doi:10.1016/j.gie.2010.07.047

[2] Khashab M, El Zein M, Sharzehi K et al. EUS-guided BD or enteroscopyassisted ERCP in patients with surgical anatomy and biliary obstruction: an international comparative study. Endosc Int Open 2016; 04: E1322-E1327

[3] Giovannini M. EUS-guided hepaticogastrostomy. Endosc Ultrasound 2019; 8: 35

[4] Anderloni A, Fugazza A, Troncone E et al. Single-stage EUS-guided choledochoduodenostomy using a lumen-apposing metal stent for malignant distal biliary obstruction. Gastrointest Endosc 2019; 89: 69-76

[5] Park JK, Woo YS, Noh DH et al. Efficacy of EUS-guided and ERCPguided biliary drainage for malignant biliary obstruction: prospective randomized controlled study. Gastrointest Endosc 2018; 88: 277-282

[6] Bang JY, Navaneethan U, Hasan M et al. Stent placement by EUS or ERCP for primary biliary decompression in pancreatic cancer: a randomized trial (with videos). Gastrointest Endosc 2018; 88: 9-17

[7] Khan MA, Akbar A, Baron TH et al. Endoscopic ultrasound-guided biliary drainage: a systematic review and meta-analysis. New York LLC: Springer;
[8] Dhir V, Artifon ELA, Gupta K et al. Multicenter study on endoscopic ultrasound-guided expandable biliary metal stent placement: Choice of access route, direction of stent insertion, and drainage route. Dig Endosc 2014; 26: 430-435

[9] Cotton PB, Eisen GM, Aabakken L et al. A lexicon for endoscopic adverse events: report of an ASGE workshop. Gastrointest Endosc 2010; 71: 446-454

[10] Sha J, Dong Y, Niu H. A prospective study of risk factors for in-hospital mortality in patients with malignant obstructive jaundice undergoing percutaneous biliary drainage. Medicine (Baltimore) 2019; 98 : e15131

[11] Takahashi K, Tsuyuguchi T, Saiga A et al. Risk factors of ineffective drainage in uncovered self-expandable metal stenting for unresectable malignant hilar biliary strictures. Oncotarget 2018; 9: 2818528194

[12] Kanno Y, Ito K, Koshita S et al. EUS-guided biliary drainage for malignant perihilar biliary strictures after further transpapillary intervention has been judged to be impossible or ineffective. Intern Med 2017; 56: 3145-3151

[13] Nakai $\mathrm{Y}$, Isayama $\mathrm{H}$, Yamamoto $\mathrm{N}$ et al. Conversion to endoscopic ultrasound-guided biliary drainage by temporary nasobiliary drainage placement in patients with prior biliary stenting. Endosc Ultrasound 2017; 6: 323-328

[14] Panpimanmas S, Ratanachu-Ek T. Endoscopic ultrasound-guided hepaticogastrostomy for advanced cholangiocarcinoma after failed stenting by endoscopic retrograde cholangiopancreatography. Asian J Surg 2013; 36: 154-158

[15] Ardengh JC, Lopes CV, Kemp R et al. Different options of endosonography-guided biliary drainage after endoscopic retrograde cholangiopancreatography failure. World J Gastrointest Endosc 2018; 10: 99108

[16] Gupta K, Perez-Miranda M, Kahaleh M et al. Endoscopic ultrasoundassisted bile duct access and drainage: Multicenter, long-term analysis of approach, outcomes, and complications of a technique in evolution. J Clin Gastroenterol 2014; 48: 80-87

[17] Sassatelli R, Cecinato P, Lupo M et al. Endoscopic ultrasound-guided biliary drainage for malignant biliary obstruction after failed ERCP in low performance status patients. Dig Liver Dis 2019: Epub ahead of print doi:10.1016/j.dld.2019.07.009

[18] Khashab M, Van der Merwe S, Kunda R et al. Prospective internationa multicenter study on endoscopic ultrasound-guided biliary drainage for patients with malignant distal biliary obstruction after failed endoscopic retrograde cholangiopancreatography. Endosc Int Open 2016; 04: E487-E496

[19] De Cassan C, Bories E, Pesenti C et al. Use of partially covered and uncovered metallic prosthesis for endoscopic ultrasound-guided hepaticogastrostomy: Results of a retrospective monocentric study. Endosc Ultrasound 2017; 6: 329

[20] Oh D, Park DH, Song T] et al. Optimal biliary access point and learning curve for endoscopic ultrasound-guided hepaticogastrostomy with transmural stenting. Therap Adv Gastroenterol 2017; 10: 42-53

[21] Paik WH, Lee TH, Park DH et al. EUS-guided biliary drainage versus ERCP for the primary palliation of malignant biliary obstruction: a multicenter randomized clinical trial. Am J Gastroenterol 2018; 113: 987-997

[22] Lee TH, Choi JH, Park DH et al. Similar efficacies of endoscopic ultrasound-guided transmural and percutaneous drainage for malignant distal biliary obstruction. Clin Gastroenterol Hepatol 2016; 14: 10111019.e3

[23] Artifon ELA, Aparicio D, Paione JB et al. Biliary drainage in patients with unresectable, malignant obstruction where ERCP fails: Endoscopic ultrasonography-guided choledochoduodenostomy versus percutaneous drainage. J Clin Gastroenterol 2012; 46: 768-774 
[24] Han SY, Kim SO, So $\mathrm{H}$ et al. EUS-guided biliary drainage versus ERCP for first-line palliation of malignant distal biliary obstruction: A systematic review and meta-analysis. Sci Rep 2019: Epub ahead of print doi:10.1038/s41598-019-52993-x

[25] Miller CS, Barkun AN, Martel M et al. Endoscopic ultrasound-guided biliary drainage for distal malignant obstruction: a systematic review and meta-analysis of randomized trials. Endosc Int Open 2019; 7: E1563-E1573

[26] Artifon ELA, Marson FP, Gaidhane M et al. Hepaticogastrostomy or choledochoduodenostomy for distal malignant biliary obstruction after failed ERCP: Is there any difference? Gastrointest Endosc 2015; 81: 950-959

[27] Wang K, Zhu J, Xing L et al. Assessment of efficacy and safety of EUSguided biliary drainage: A systematic review. Gastrointest Endosc 2016; 83: 1218-1227

[28] Ogura T, Chiba Y, Masuda D et al. Comparison of the clinical impact of endoscopic ultrasound-guided choledochoduodenostomy and hepaticogastrostomy for bile duct obstruction with duodenal obstruction. [Erratum appears in Endoscopy. 2016 Feb;48(2):163; PMID: 26418074]. Endoscopy 2016; 48: 156-163

[29] Sharaiha RZ, Khan MA, Kamal F et al. Efficacy and safety of EUS-guided biliary drainage in comparison with percutaneous biliary drainage when ERCP fails: a systematic review and meta-analysis. Gastrointestinal Endoscopy 2017; 85: 904-914

[30] Sportes A, Camus M, Greget M et al. Endoscopic ultrasound-guided hepaticogastrostomy versus percutaneous transhepatic drainage for malignant biliary obstruction after failed endoscopic retrograde cholangiopancreatography: A retrospective expertise-based study from two centers. Therap Adv Gastroenterol 2017; 10: 483-493

[31] Bapaye A, Dubale N, Aher A. Comparison of endosonography-guided vs. Percutaneous biliary stenting when papilla is inaccessible for ERCP. United Eur Gastroenterol J 2013; 1: 285-293

[32] Ogura T, Onda S, Takagi W et al. Clinical utility of endoscopic ultrasound-guided biliary drainage as a rescue of re-intervention procedure for high-grade hilar stricture. J Gastroenterol Hepatol 2017; 32: 163-168

[33] Minaga K, Takenaka M, Kitano M et al. Rescue EUS-guided intrahepatic biliary drainage for malignant hilar biliary stricture after failed transpapillary re-intervention. Surg Endosc 2017; 31: 4764-4772

[34] Paik WH, Lee NK, Nakai Y et al. Conversion of external percutaneous transhepatic biliary drainage to endoscopic ultrasound-guided hepaticogastrostomy after failed standard internal stenting for malignant biliary obstruction. Endoscopy 2017; 49: 544-548

[35] Park DH, Lee TH, Paik WH et al. Feasibility and safety of a novel dedicated device for one-step EUS-guided biliary drainage: A randomized trial. J Gastroenterol Hepatol 2015; 30: 1461-1466

[36] Paik W, Park D. Outcomes and limitations: EUS-guided hepaticogastrostomy. Endosc Ultrasound 2019; 8: 44

[37] Tyberg A, Desai AP, Kumta NA et al. EUS-guided biliary drainage after failed ERCP: a novel algorithm individualized based on patient anatomy. Gastrointest Endosc 2016; 84: 941-946 